\title{
The Application of ATCA Structure for Power Communication Switching System
}

\author{
HaiQing Yin \\ State Grid Electric Power Research Institute \\ Nanjing, 210000, China \\ E-mail:pp19871002pp@163.com \\ JingHong Guo \\ China Electric Power Research Institute \\ Nanjing, 210000, China \\ E-mail: guojinghong@epri.sgcc.com.cn
}

\author{
WeiNing WU \\ State Grid Electric Power Research Institute \\ Nanjing, 210000, China \\ E-mail: wuweining@sgepri.sgcc.com.cn \\ JunMin Wu \\ China Electric Power Research Institute \\ Nanjing, 210000, China \\ E-mail : wujunmin@epri.sgcc.com.cn
}

\begin{abstract}
In order to meet the needs of the power communication device This paper discusses the key technologies of the power switching equipment based on ATCA structure, including network topology selection and rack management system, as well as a system performance test result is given. The test results show that the power switching equipment based on ATCA structure has good rack management ability, and it can monitor and adjust parameters such as system temperature. It can realize hot backup of the system, making the system with high reliability and high transmission bandwidth.
\end{abstract}

\section{INTRUCTION}

Power communication network comes into being in order to guarantee the safe and stable operation of the power system, and it is base of building smart grid. With future grid dispatching automation, network operators, market-oriented, and management of modern development, power communication network is a more important mean to ensure grid security, stability and economic operation, and it is the critical infrastructure of the power system. The future smart grid puts forward higher requirements for power communication network reliability, transmission bandwidth, flexibility and anti-disaster.

Power communication network transmission services include the following four points:

1) Transmission of smart electricity transmission information, including line status, tower status, weather condition.

2) Transmission of smart substation information, including a device status, secondary equipment status.

3) Transmission of smart power distribution Information, including distribution status, power quality.

4) Transmission of smart use of electricity Information, including measurement assessment, the electricity consumption of the residents.

As it is shown above, the main task of the power communication system is to transmit the physical data which includes remote measurement, remote information, remote control and remote adjustment of the power system.

\section{DEMAND OF FUTURE SMART GRID FOR COMMUNICATION EQUIPMENT}

Demand of future smart grid for communications equipment includes: high-reliability, high-bandwidth, realtime, open, standardized and integrated.

High reliability: With the construction of UHV grid, grid stability, security and economic operation put forward higher requirements. In some cases, the working environment and electromagnetic environment is poor. When the communication device is close to the power line, Electromagnetic interference and lightning affection is especially serious. It must have strong anti-interference and good electromagnetic compatibility requirements in order to ensure the low error rate of data transmission, and the equipment can work reliably for a long time in the harsh environment. [1] This device has a redundant backup feature, good resistance and sound system control mechanisms.

High-bandwidth and real-time: With the building and promoting of the grid panoramic visualization, the company SG-ERP and data disaster recovery center, power communications business will have higher bandwidth requirements during "Twelfth Five-Year" period. There will be a large amount of video and data services carried by electric power communication network; what's more the business systems and applications tend to be together. It needs higher requirements for capacity of the business carrying and performance of electric power communication network especially for data communication network.[2] Power communication network is a nervous system in order to connect every perception terminal and collection terminal of the grid, and it can realize information transmission and "perception" of the grid [3]. In order to monitor the state of the related equipment, find and isolate faults quickly by equipment of measurement and protection, it need process information fast and real-time.

Open and standardization: With the development of the smart grid in the future, number of smart power devices will continue to increase in order to be able to get the grid panoramic information. Smart devices need to be compatible with the other device such as parameters, status and control commands. Building open communication architecture to forming a "plug and play" environment can realize sharing substation resource information, interoperability of smart devices and simplifying system maintenance and 
configuration. Majority of device manufacturers invent devices which use bus and plug, and it is of some scalability. But the custom backplane bus and plug-ins do not follow the same international standards, and it limits versatility between the various manufacturers devices, the device can't achieve interchangeable in plug-grade. [1]

Integration: Future grid requires intelligent electronic devices and control centers communicate by network. So it can lead data and information centralized collection and transmission. It can achieve a high degree of integration and sharing of grid information. However, the number of existing substation equipment is big, and integration is poor. What's more wiring between devices is complex which leads to poor reliability. Due to the hardware principle and operation of each device vary greatly, maintenance workload is high. Operation and maintenance need many professionals to cooperate, and it may increase probability of errors and reduce reliability.

\section{Selection Of The Bus Structure}

In order to meet need of the future smart grid for communication equipment which is of high reliability, highbandwidth, real-time, open, standardization and integration requirements, it is necessary to select a advanced and standardized bus architecture to design power communication equipment.

Universal computer bus architectures include VEM, CPCI, PXI and ATCA.

VME (VersaModule Eurocard) bus is a universal computer bus which combines Versa bus electrical standards of the Motorola Company and Eurocard in Europe standard, and it is an open architecture.

CPCI bus (Compact PCI) is a high-performance industrial computer bus standard which is proposed by PICMG (PCI Industrial Computer Manufacturer's Group, the international industrial computer manufacturer Federation) in 1994.

PXI completed the development in 1997 and launched in 1998 officially, and it is based on the PCI (Peripheral Component Interconnect),Compact PCI and some combination of PXI-specific signals.

ATCA bus (Advanced Telecom Computing Architecture) is the most significant innovations in PICMG (PCI Industrial Computer Manufacturer's Group) standard history, and it is approved in December 2002.

\begin{tabular}{|c|c|c|c|c|}
\hline 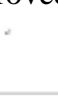 & $\begin{array}{l}\text { functional } \\
\text { density of } \\
\text { the rack. }\end{array}$ & $\begin{array}{l}\text { Transmission } \\
\text { bandwidth }\end{array}$ & reliability & Open,standardized. \\
\hline VEM & low & $160 \mathrm{M} / \mathrm{s}$ & high- & $\begin{array}{l}\text { Not compatible } \\
\text { with pci bus. }\end{array}$ \\
\hline $\mathrm{CPCl}$ & low. & $512 \mathrm{M} / \mathrm{s}$ & high & high \\
\hline PXL. & low & $528 \mathrm{M} / \mathrm{s}$ & high & high \\
\hline ATCA. & high & $10 \mathrm{G} / \mathrm{s}$ & high & high \\
\hline
\end{tabular}

Figure 1. Comparison of Various Bus Architecture

ATCA has a high rack density (each card can accommodate more integrated circuit chips), high transmission bandwidth and reliability, and it is open and standardized, what's more it has standard intelligent management interface.

So it is able to meet the needs of the power communication device. So this article chooses ATCA as the architecture of the electric power communications equipment.

\section{System DESIGN POINTS}

\section{A. Selection Of the Network Structure}

PICMG3.0 can support three kinds of network structures: double-star, double - double-star and full-mesh structure. However, these three kinds of structures have own advantages and disadvantages:

- double-star

Advantages: 1. each board and the central switching board (main and backup) have a connection to improve reliability by redundancy. 2 . It is relatively easy to find fault, and if a board fails, it is easy isolate the board from the entire system. 3 It is relatively easy to expand, and new business cards can be easily added to the chassis inside.

Disadvantages: 1 . Whether a system can work properly depends on the central exchange board relationship greatly. Once it fails, the entire system will not work properly. 2 . The entire system of network traffic go through the central exchange board, and it will lead a relatively large burden which is likely to cause the network congestion.

- Double - double-star

The advantages and disadvantages is almost same as double-star, and the only difference is hardware redundancy. Double - double-star has two double-star structure which increases reliability than the double-star. But it also increases the cost inevitably.

- Full mesh structure:

Advantages: 1 . Network reliability is high, and there are two or more communication paths between any two nodes. 2 . Network configuration is easy, and it can choose a variety of transmission rate. 3 . Resource can be shared between nodes easily. 4. It can improve line flow distribution. 5. It can choose the best path to achieve smaller transmission delay.

Disadvantages :

1 The software structure and operation is complex.

2 Connection is increased, and wiring becomes complicated which lead the PCB layers and the cost to be increased.

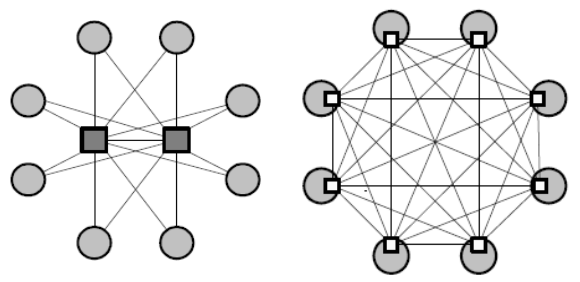

Figure 2. dual-star and full-mesh structure

Take the cost, reliability and difficulty to develop into consideration, and this project chooses the Double Star redundant structure .So each plate has a channel with two different exchange board, and it can achieve a more reliable and real-time network traffic. 


\section{B. Rack Management}

The rack management components mainly consist of two parts (Figure 3): two separate mutual hot backup ShMC (shelf manage control) and IPMC which is located in each business board. ShMC is responsible for managing the entire rack equipment. IPMC is used to contact with ShMC, and another effect is to manage its own board.

System management is performed by ShMC. ShMC is responsible to manage the FRU(Field Replaceable Units) of the ATCA system such as: veneer, power supply, fan, temperature sensor, hot-swappable and veneer interface type.

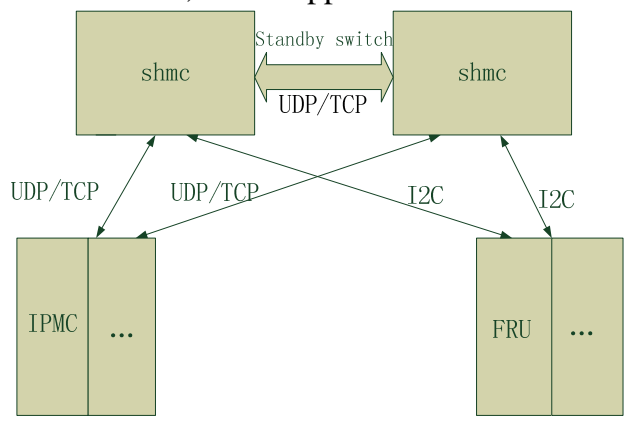

Figure 3. Rack Management Structure

- Channel between ShMC and FRU

ShMC controls FRU by I2C bus. I2C is a two wire serial interface which has data and clock. It uses $100 \mathrm{Khz}$ clock and 3.3V signaling. The I2C bus is used to connect the FRU and ShMC. The specific design is shown in Figure 4:

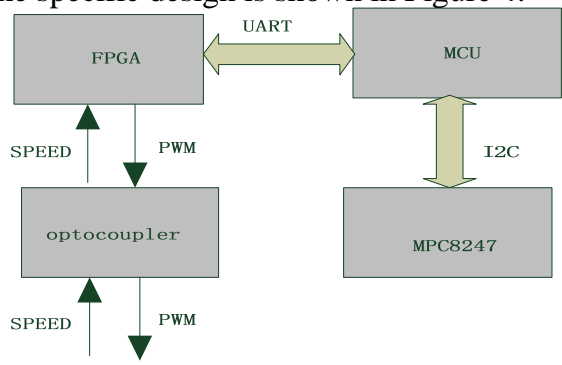

Figure 4. Specific Design

FPGA: FPGA is used to control the fan. One hand, the microcontroller obtains control information of the fan by the UART, then it generates PWM l signal to control the fan by the optocoupler; On the other hand, FPGA samples the fan speed signal and reports to the microcontroller by the UART. FPGA chooses XC3S50AN-4TQG144-I mode.

MCU: MCU connects FPGA and the main control board (via the backplane) by UART and I2C interface separately. On one hand, the microcontroller reports fan speed to the main control board by the I2C interface, and at the same time it get the instruction of the control board for the fan speed.I2C interface is separated by the hot-swap chip LTC4300. On the other hand, the microcontroller gets fan information from the FPGA by the UART .And it generate the PWM signal by FPGA as strategy which is given by the main control board.
MPC8247: MPC8247 connected microcontroller by I2C interface. MPC8247 transmits control information to MCU by the I2C as the strategy given by the application. Then microcontroller transmits information to the FPGA by UART, and FPGA controls the fan speed by changing the duty cycle of the PWM wave.

- Channel between ShMC and PMC

ShMC controls IPMC (Figure 5) by UDP / TCP transmission, using 100BASE-T level, and signal uses two pairs of differential signal in backplane base area. It is recommended to choose Marvell 88E6095 as switch chip, and Marvell 88E6095 has $8 \mathrm{FE}$ ports and three GE ports. When IMPC starts, it opens two client and tries to connect the ShMC(Active and Standby boar). If ShMC starts, ShMC which is on will give the response to IMPC, and the state of IMPC is on. Since then, IMPC builds a asynchronous event channel (report client), and responds to RPC call launched by ShMC. ShMC establish a socket connection with IMPC. It can obtain the IMPC the connection status timely, and it can notice IMPC When ShMC occurs standby switching

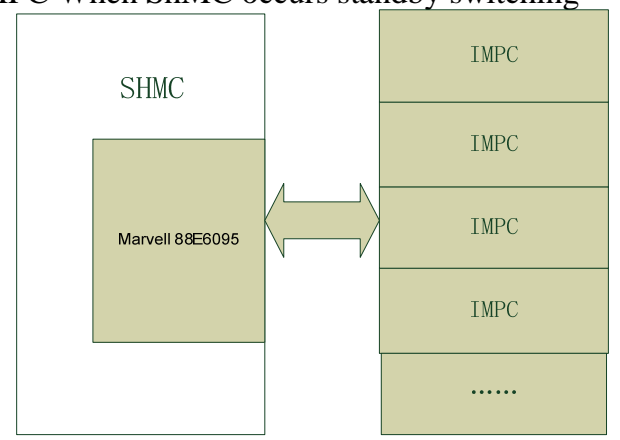

Figure 5. Management Principle

- Channel between ShCMs

ShCM communicates with ShCM by UDP/TCP to realize standby switch, and it can ensure the reliability of the system. When the backup plate can't receive the valid signal of the primary system within the specified time, the backup board will take over the system. This is mainly realized by hot backup interlock signal to pass heartbeat messages.

Heartbeat packet can be transmitted by marvell switch chip, and it can also be transmitted by broadcom switch chip. Because marvell switch chip is used to transmit the control information (data flow is low).But broadcom switch chip is used for the transmission of business data (data flow is high), it may lead the heartbeat packets can't be received correctly, so the device selects marvell switch chip to transmit Heartbeat packet.

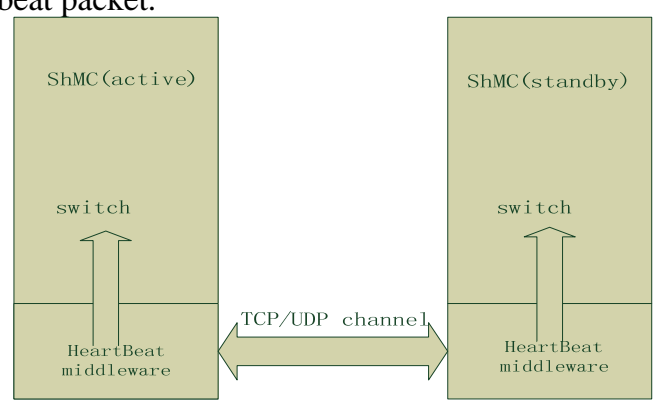


Figure 6. standby switch

\section{System TEST}

- Test Of Fan Control

Test steps:

1. Use the command "show fan info" to View fan status, and use the command "show fan speed parameter" to view fan control parameters.

2.Use the Command "speed mode manual" to set fan to manual mode, use the command "fan speed level $<1-4>$ "to Set the fan speed level and detect whether the speed is normal at different temperatures.

3. Use the command "fan speed mode auto" to set the fan to auto mode, and use the command "fan speed policy $<1-4>$ ”to set the fan speed and detect whether the speed is normal at different temperatures

Test results: fan control is normal.

- System Throughput test

1. System consists of 12 IMPC and 2 ShCM .IPMC is in pairs connected by ONU. The first group ONU and the last piece of IMPC are connected by Network Tester.

2. Adjacent PON of two IPMCs is divided as a VLAN.

3. Using a network tester system throughput of $90 \%$ (overloaded) test. The test frame length, respectively $64,128,256,512,1024,1280,1518$, testing time is 120 s.

Observe network tester packet loss rate.

Test results: 120s no packet loss.

- Standby Switch Function Test, test steps:

1 Follow image to build a test environment (Figure 7);

2 Network Tester $100 \%$ of the system throughput (overloaded) test, the test frame length respectively $64,128,256,512,1024,1280,1518$. The ONU1 flow is $400 \mathrm{Mbps}$. The ONU2 flow is $400 \mathrm{Mbps}$.The ONU3 flow is $400 \mathrm{Mbps}$.And the flow is uninterrupted.

3 Observe the Network Tester packet loss rate;

4 Login ShMC board (active) by the command line, and view the system configuration.

5.Unplug ShMC board (active) during the system throughput test.

6 View the record of the main switchover function.

Test results:

1 Switching time is less than or equal to 8S.

2 Standby control board switches to ACTIVE;

3 , the command line is working properly;

As can be seen from the above test results, power switching equipment based on the ATCA architecture can meet the business needs.

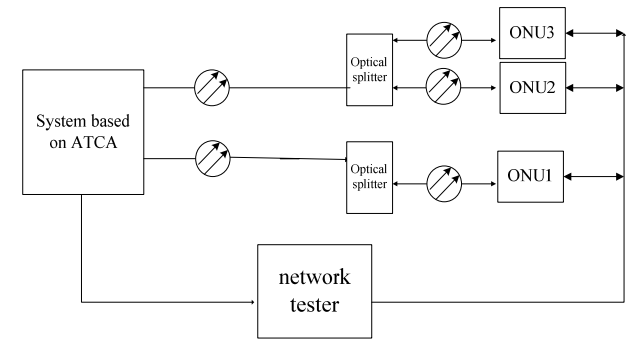

Figure 7. Test Environment

\section{SUMMARY}

This paper studies the key technology of the ATCA framework for power exchange system: network structure and rack management system. As the development of the smart grid, power system will propose higher requirements on the reliability and transmission bandwidth of electric power communication equipment.

ATCA structure can help the equipment to improve the data processing capacity and speed of data transmission. It provides a sufficient number of the external connection interface and the safe and efficient system management mechanism. What's more it has a standard and reliable system connection. It is easy to maintain the system structure, good openness, resistance to harsh environments and fault tolerance features. ATCA structure will have a very wide range of applications in power communication device.

REFERENCES

[1] Y.G. Wang, J. Mao ,Strong for a the CPCI bus-based intelligent fault information system sub-station ,power System Protection and Control 201038 (11)

[2] Z.Y. Zhao, D.W.Zhang, State Grid Corporation of "second five" power communications business requirements analysis, Power System Communication 201132 (223)

[3] X. Chen , X. Miao and J. Zhou ,Development strategy of slectric power systems, Power system Communication 201132 (223)

[4] Z.X. Liang, J. Wang and W.L. Tang, The EPON technology applied research and network design in smart distribute grid, PowerSystem Communication 201233 (232)

[5] G.J. Gong, Y. Sun andM.M. Cai Physical network architecture for smart grid applications research, Power System Protection and Control 201139 (20)

[6] L. Zhao Smart grid key IT decision support, Sun Yat-sen University master's degree thesis

[7] X. Gao The digital substation application technology, Beijing,China ,Electric Power Press, 2008.

[8] H.J. Zhangdigitized Substation integrated information platform,Power Automation 2008.10

[9] J. Zhang ,B. Song, Protection and monitoring device the embedded sampling a new platform for power system automation ,Power System Automation 2011.1

[10] G.F Huang,S.M. Xu, Key technology of digital substation protection and monitoring device ,Jiangsu Electrical Engineerin 